\title{
Exact Evaluation of Statistical Moments in Superradiant Emission
}

\author{
Gilberto M. Nakamura ${ }^{1}\left(\mathbb{D}\right.$, Brenno Cabella $^{2}\left(\mathbb{D}\right.$ and Alexandre S. Martinez ${ }^{2,3, *}$ \\ 1 Faculdade de Filosofia, Ciências e Letras de Ribeirão Preto (FFCLRP), Universidade de São Paulo (USP), \\ Avenida Bandeirantes 3900, 14040-901 Ribeirão Preto, São Paulo, Brazil; gmnakamura@usp.br \\ 2 Instituto de Física Teórica, Universidade Estadual Paulista (UNESP), Rua Dr. Bento Teobaldo Ferraz 271, \\ 01140-070 São Paulo, Brazil; cabellab@gmail.com \\ 3 Instituto Nacional de Ciência e Technologia de Sistemas Complexos (INCT-SC), Rua Dr. Xavier Sigaud 150, \\ Urca, 22290-180 Rio de Janeiro, Brazil \\ * Correspondence: asmartinez@usp.br
}

Received: 30 April 2019; Accepted: 19 June 2019; Published: 23 June 2019

\begin{abstract}
Superradiance describes the coherent collective radiation caused by the interaction between many emitters, mediated by a shared electromagnetic field. Recent experiments involving Bose-Einstein condensates coupled to high-finesse cavities and interacting quantum dots in condensed-matter have attracted attention to the superradiant regime as a fundamental step to create quantum technologies. Here, we consider a simplified description of superradiance that allows the evaluation of statistical moments. A correspondence with the classical birthday problem recovers the statistical moments for discrete time and an arbitrary number of emitters. In addition, the correspondence provides a way to calculate the degeneracy of the problem.
\end{abstract}

Keywords: stochastic processes; complex systems; self-organization; Dicke model; birthday problem

\section{Introduction}

Superradiance describes the coherent collective radiation caused by the interaction between $N$ emitters, mediated by a shared electromagnetic field [1]. The phenomenon occurs when the average distance between emitters is smaller than the emitted wavelength, producing an emission pattern that differs markedly from the spontaneous emission of photons by isolated atoms. Supporting experimental evidence shows that the electric dipole of atoms in the atomic cloud becomes strongly correlated, producing coherent radiation less sensitive to thermal fluctuations of optical cavities [2-4], which can be used to improve the precision of atomic clocks [5]. Dicke first predicted superradiance after considerations on symmetries and conservation laws of total angular momentum in matter-light interaction in cavities. Almost 70 years after its discovery, superradiance remains a topic of intense experimental and theoretical research in quantum many-body physics [6,7].

Although its origins can be tracked down to the foundation of quantum optics, superradiance has found applications in condensed-matter physics. More specifically, the experimental observation of superradiance in ensembles of quantum dots suggests long-range electromagnetic interactions can be fine-tuned for creating quantum technologies, by selecting an appropriate density of quantum dots, their physical dimensions, and their atomic composition or the surrounding media [8]. The iconic spontaneous mission of photons by emitters may not occur as $N$ independent events, even for $N=2$, as verified in recent experiments [9]. Enhanced spontaneous emission can also occur due to strong correlations between quantum dots immersed in confining potentials [10]. More broadly, theoretical and experimental studies of superradiance in condensed-matter have led to reexamination of collective effects in nanoscale systems [11,12], metallic nanoparticles and nanostructures [13,14], and magnetic nanosystems, as well [15-18]. 
Meanwhile, experimental realizations of the superradiant regime in Bose-Einstein condensates coupled with optical cavities have provided insights into quantum phase transition between normal and superradiant phases $[19,20]$. In these experiments, the atomic condensate remains trapped inside a high finesse optical cavity. A continuous wave with the frequency far detuned from relevant atomic transition is pumped onto the condensate, transverse to the axis of the optical cavity. Because the pump wave is detuned, the interaction is dispersive with negligible spontaneous emission. As a result, waves are scattered into the cavity with resonant frequency $\omega$, which self-organize the condensate in a spatial lattice, with half-wavelength spacing. Even more, the resulting lattice acquires one out of two possible orientations, suggesting a $\mathbb{Z}_{2}$ spontaneous symmetry-breaking at the quantum phase transition [21]. Indeed, a phase transition in the Dicke model has been predicted by Hepp and Lieb [22]. The Hamiltonian of the Dicke model reads:

$$
\hat{H}=\hbar \omega \hat{a}^{\dagger} \hat{a}+\hbar \omega_{0} \hat{J}_{z}+\frac{\gamma}{\sqrt{N}}\left(\hat{a}^{\dagger}+\hat{a}\right) \hat{J}_{x}
$$

formed by $N$ two-level emitters with energy $\hbar \omega_{0}$ and a single bosonic mode with cavity frequency $\omega$ and coupling $\gamma$. The collective pseudospin operators $\hat{J}_{\mu}(\mu=x, y, z)$ satisfy the usual relations $\left[\hat{J}_{\alpha}, \hat{J}_{\beta}\right]=$ $i \hbar \sum_{\mu} \varepsilon_{\alpha \beta \mu} \hat{J}_{\mu}$. The light-matter interaction is described by the operator $\left(\hat{a}^{\dagger}+\hat{a}\right) \hat{j}_{x}=(1 / 2)\left(\hat{a}^{\dagger} \hat{J}_{-}+\right.$ $\left.\hat{a} \hat{J}_{-}\right)+(1 / 2)\left(\hat{a}^{\dagger} \hat{J}_{+}+\hat{a} \hat{J}_{-}\right)$. This operator carries two familiar transitions, namely the emission of a photon to the cavity and the photon absorption, as well as two apparently non-conservative energy transitions: photon emission followed by atomic excitation and photon absorption followed by atomic relaxation. They appear non-conservative because pump waves are not taken into account, resulting in an open system. Finally, the intensity I produced by a superradiant system contains contributions proportional to $\left|\left\langle j, m_{z} \pm 1\left|\hat{J}_{ \pm}\right| j, m_{z}\right\rangle\right|^{2}=j(j+1)-m(m \pm 1)$. For $j=N / 2$, transitions starting from quantum states with $m_{z}=0$ maximize the emission of radiation and produce $I \propto N^{2}$, which is one of the hallmarks of superradiance. The intensity diminishes for other values of $\left|m_{z}\right|$, becoming a linear function of $N$ for $\left|m_{z}\right|=j=N / 2$.

To date, Bose-Einstein experiments have offered the most flexibility to select couplings and explore the superradiant phase transition and its critical properties in detail. Among them is the emergence of long-range correlations among emitters, which can be further explored to create faster procedures for information storage and retrieval in qubit networks [23]. The key aspect to understand the underlying physics in the Dicke model is the proper comprehension of self-organizing phenomena [12]. The ordered collective behavior arises from small fluctuations and develops positive feedback [24-29]. These collective effects are observable and reveal the effect of higher order statistical moments and correlation functions. In the Dicke model, several approaches have been proposed to capture the minimal cooperative properties. In the thermodynamic limit $(N \gg 1)$, the model has been studied in the rotating wave approximation (RWA) [22,30]. More recently [31], without RWA and using the Holstein-Primakoff transformations with $N \gg 1$, Emary and Brandes presented an exact solution, where they verified the existence of quantum phase transitions and the emergence of a chaotic regime. For finite $N$, the interference among confined emitters is enhanced by finite size effects, and the model is non-integrable [32], while the available solutions are restricted to numerical ones [33,34].

More recently, semiclassical approximations have provided a far more concrete structure of the density of states of the Dicke model, including evidence of excited-state quantum phase transitions $[7,35]$. These advances also include microcanonical calculations and thermodynamic properties [36]. However, a complete picture of the quantum problem is still lacking. Alternatively, one can probe the properties of superradiant systems using inferences from stochastic processes. While limited in scope, the purpose of simplified models is to produce insights into specific aspects of the dynamics of superradiance in a more tractable manner [37]. Here, we address the evaluation of statistical moments and degeneracy in superradiance by considering a correspondence between a self-organizing process, which mimics the superradiance, and the well-known birthday problem. This connection allows one to study the degeneracy in both a small and large number of emitters and, 
thus, the aspects of the superradiant dynamics. The outline of the paper is as follows. In Section 2, we introduce a self-organizing process, in which a small initial fluctuation gives rise to the rapid growth of photon numbers in the cavity. The existing symmetries are identified, and analytic expressions for the $\ell^{\text {th }}$ statistical moments are derived. In Section 3, a correspondence between the self-organizing process and the birthday problem is unveiled allowing the exact evaluation of the $\ell^{\text {th }}$ statistical moment beyond the Poisson approximation (arbitrary time) and small N. We present a Monte Carlo example and compare its results to our exact calculations. Our closing remarks are listed in Section 4.

\section{Superradiance}

At its core, a superradiant pulse mimics a cascade of photon emission from a population composed of excited emitters. The complexity of the phenomenon arises from the coupling with a shared radiation field: the ensemble of $N$ emitters can only create a single photon per unit of time, according to Equation (1). The constraint introduces temporal correlations among the emitters that dictate the collective decay. Here, we consider a self-organizing system formed by $N$ emitters in a resonant cavity. Each emitter has two levels, and they are located close enough to each other to interfere through the common radiating field. At a given time instant $t$, there are $n(t)$ emitters in their respective ground states and $N-n(t)$ excited emitters. The self-organizing constraint is imposed by stating that no more than one emission occurs during a time interval $\delta t$, i.e., photon emission events are not independent. The emission process is composed by two stochastic events. A single emitter among $N$ available is selected with uniform probability $p=1 / N$. If the selected emitter is found in the excited state, the subsequent emission occurs with conditional probability $p_{e}=1$, otherwise $p_{e}=0$. This stochastic process ensures that the total number of excited states can only be decremented by one for successive time steps. The assumption of uniform $p$ implies that emitters are equally affected by the radiation field. It oversimplifies the spatial distribution of emitters in atomic clouds, excluding superradiant emission due to non-linear effects [37]. A naive analysis of the collective probability distribution, ignoring the superradiant constraint and thus correlations among emitters, leads to a Poissonian distribution. This is not the case, as we show in what follows.

Let $|n\rangle$ represent the configuration containing $n$ emitters in the ground state in an ensemble with $N$ emitters. The stochastic process compromises the transitions that occur along time on the vectors $|n\rangle$. The transitions are encoded by the transition matrix $\hat{T}$, which reads:

$$
\hat{T}=\left(\begin{array}{cccccc}
0 & 0 & 0 & & 0 & 0 \\
1 & p & 0 & \cdots & 0 & 0 \\
0 & 1-p & 2 p & & 0 & 0 \\
& \vdots & & \ddots & \vdots & \\
0 & 0 & 0 & \cdots & 1-p & 0 \\
0 & 0 & 0 & & p & 1
\end{array}\right),
$$

as illustrated in Figure 1. The operator $\hat{T}$ possesses some notable properties: $\operatorname{Tr}(\hat{T})=(1+p) / 2$, and $\sum_{\mu=0}^{N} T_{\mu \nu}=1$ ensures probability conservation. In addition, $\hat{T}$ is triangular with a clear pattern for each occupation level $n$. The analogy with angular momentum algebras leads to:

$$
\hat{T} \equiv p\left(\hat{J}_{z}+j \hat{1}\right)+\hat{J}_{+},
$$

where $\hat{1}$ is the identity operator, $n=m_{z}+j\left(j=0,1, \ldots, N / 2\right.$ and $\left.m_{z}=-j,-j+1, \ldots, j\right), \hat{J}_{+}|n\rangle=$ $(1-p n)|n+1\rangle$. Accordingly, $\left[\hat{J}^{2}, \hat{T}\right]=0$, and $j$ is a conserved quantity. The eigenvalue $j$ is set by the initial conditions: for $N$ emitters in the excited state, $j=N / 2$.

Once the transition matrix is defined, one writes the master equation:

$$
\partial_{t}|P(t)\rangle=-\hat{\mathcal{H}}|P(t)\rangle,
$$


in which $\hat{\mathcal{H}} \equiv(\hat{1}-\hat{T}) / \delta t$ is the generator of temporal translations, with a role similar to the Hamiltonian in quantum systems. The probability vector $|P(t)\rangle=P_{0}(t)|0\rangle+P_{1}(t)|1\rangle+\cdots+P_{N}(t)|N\rangle$ is a linear combination of occupation vectors, and the coefficients $P_{n}(t)$ describe the instantaneous probability to measure $n$ emitters in the ground state. The coefficients $0 \leqslant P_{n}(t) \leqslant 1$ satisfy $\sum_{n=0}^{N} P_{n}(t)=1$. The eigenvalues of $\hat{\mathcal{H}}$ are readily available, $E_{n}=(1-p n)$, with $n=0,1, \ldots, N$, but the $\hat{\mathcal{H}}$ is not Hermitian. As a result, right and left eigenvectors, respectively $\left|\phi_{k}\right\rangle$ and $\left\langle\chi_{k}\right|$, are not related by Hermitian conjugation. The eigenvectors obey the identity decomposition, $\sum_{k=0}^{N}\left|\phi_{k}\right\rangle\left\langle\chi_{k}\right|=\hat{1}$, and are orthogonal to each other. Using the spectral decomposition, the solution of the stochastic problem reads $|P(t)\rangle=\sum_{n=0}^{N} c_{n} \mathrm{e}^{-E_{n} t}\left|\phi_{n}\right\rangle$, with $c_{n}=\left\langle\chi_{n} \mid P(0)\right\rangle$. The mode with vanishing eigenvalue $E_{N}$ describes the asymptotic solution or stationary state, corresponding to all emitters in their respective ground states.

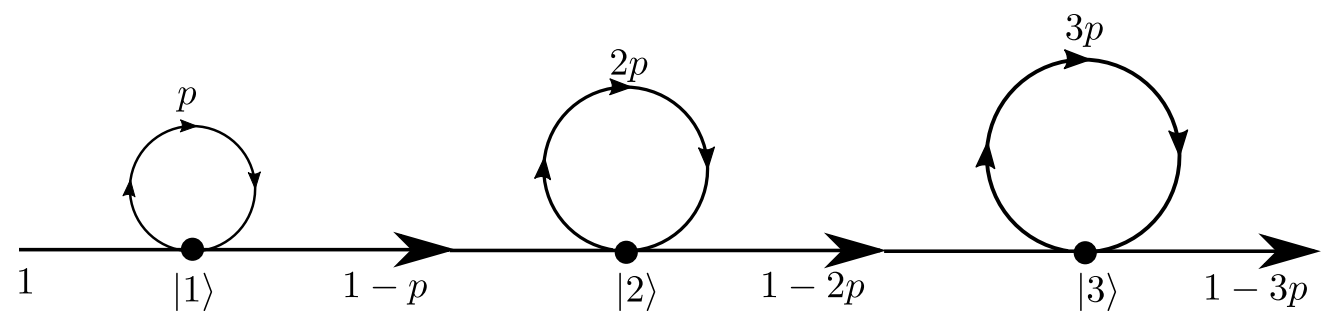

Figure 1. Superradiant stochastic process. The picture illustrates the transition $|n\rangle \rightarrow|n+1\rangle$ (horizontal arrows), with conditional probability $P(n+1, t+\delta t \mid n, t)=1-n p$, and the transition $|n\rangle \rightarrow|n\rangle$ (loops), $P(n, t+\delta t \mid n, t)=n p$. States with higher occupancy rates $n \gg 1$ have lower chances of radiating and spend more time in the same configuration. This process can be identified as the classical coupon collector's problem.

We propose an educated guess for left and right eigenvectors: there is a one-to-one correspondence between the coefficients of $\left\langle\chi_{n}\right|$ and the coefficients of the Pascal triangle of order $N$. Note that it is necessary to calculate only one set of eigenvectors, say $\left\{\left\langle\chi_{n}\right|\right\}$, because the remaining eigenvectors can be calculated via $\sum_{k=0}^{N}\left|\phi_{k}\right\rangle\left\langle\chi_{k}\right|=\hat{1}$. Surprisingly, the coefficients of $\left|\phi_{n}\right\rangle$ are also related to the Pascal triangle. For instance, the eigenvalues and respective right (left) eigenvectors are found in Table 1 (Table 2) with $N=4$. The initial probability vector $|P(0)\rangle=|0\rangle$ acquires the following decomposition:

$$
|P(0)\rangle=\sum_{n=0}^{N}\left(\begin{array}{l}
N \\
n
\end{array}\right)\left|\phi_{n}\right\rangle
$$

Using this result, we calculate the $\ell^{\text {th }}$ moment of the occupation number:

$$
\left\langle n^{\ell}(t)\right\rangle=\mathrm{e}^{-t} \sum_{k=0}^{N} \sum_{n=k}^{N} \mathrm{e}^{(k / N) t}\left(\begin{array}{l}
N \\
k
\end{array}\right)\left(\begin{array}{c}
N-k \\
n-k
\end{array}\right)(-1)^{n+k} n^{\ell} .
$$

so that the first statistical moment is $\langle n(t)\rangle=N\left(1-\mathrm{e}^{-t / N}\right)$. As expected, the system converges exponentially to a stationary state with a characteristic time scale $\tau=N$. Similarly, the second moment is evaluated,

$$
\frac{\left\langle n^{2}(t)\right\rangle}{N^{2}}=1-\left(2-\frac{1}{N}\right) \mathrm{e}^{-t / N}+\left(1-\frac{1}{N}\right) \mathrm{e}^{-2 t / N},
$$

while the standard deviation per emitter is:

$$
\frac{\sigma}{N}=\frac{\mathrm{e}^{-t / 2 N}}{\sqrt{N}}+o\left(\mathrm{e}^{-3 t / 2 N}\right) .
$$


Table 1. Right eigenvectors $\left|\phi_{k}\right\rangle=\sum_{n=0}^{N} d_{n}^{(k)}|n\rangle$ of $\hat{\mathcal{H}}$ with $N=4$. The first row displays the eigenvalues $E_{k}$, while columns display the coefficients $d_{n}^{(k)}$ in the basis $|n\rangle$. Observe the combinatorial pattern in each column $d_{n}^{(k)}=(-1)^{k+n}(N-k) ! /(n-k) !(N-n)$ ! for $n \geq k, 0$ otherwise.

\begin{tabular}{rrrrrr}
\hline $\boldsymbol{E}$ & $\mathbf{1}$ & $\mathbf{3} / 4$ & $\mathbf{2 / 4}$ & $\mathbf{1 / 4}$ & $\mathbf{0}$ \\
\hline$d_{0}$ & 1 & & & & \\
$d_{1}$ & -4 & 1 & & & \\
$d_{2}$ & 6 & -3 & 1 & & \\
$d_{3}$ & -4 & 3 & -2 & 1 & \\
$d_{4}$ & 1 & -1 & 1 & -1 & 1 \\
\hline
\end{tabular}

Table 2. Left eigenvectors $\left\langle\chi_{k}\right|=\sum_{n=0}^{N}\langle n| q_{n}^{(k)}$ of $\hat{\mathcal{H}}$ with $N=4$. The first column displays the eigenvalues $E_{k}$, while rows display the coefficients $q_{n}^{(k)}$ in the basis $\langle n|$.

\begin{tabular}{cccccc}
\hline $\boldsymbol{E}$ & $\boldsymbol{q}_{\mathbf{0}}$ & $\boldsymbol{q}_{\mathbf{1}}$ & $\boldsymbol{q}_{\mathbf{2}}$ & $\boldsymbol{q}_{\mathbf{3}}$ & $\boldsymbol{q}_{\mathbf{4}}$ \\
\hline 1 & 1 & & & & \\
$3 / 4$ & 4 & 1 & & & \\
$2 / 4$ & 6 & 3 & 1 & & \\
$1 / 4$ & 4 & 3 & 2 & 1 & \\
0 & 1 & 1 & 1 & 1 & 1 \\
\hline
\end{tabular}

The crucial step that leads to Equation (6) is counting degenerate states derived from ordered permutations of $N$ distinct objects. To do so, we consider the ordered time evolution (OTE) vector $\left|\psi\left(t_{\kappa}\right)\right\rangle \equiv\left|l_{1} l_{2} \cdots l_{\kappa}\right\rangle$, at discrete time $t_{\kappa}$. For the sake of convenience, $t_{\kappa}=\kappa \delta t$. Here, $l_{j}=1,2, \ldots, N$ identifies a single emitter selected at time $t_{j}$, with $j=1,2, \ldots, \kappa$. In this way, $\left|\psi\left(t_{\kappa}\right)\right\rangle$ is just a list containing all emitters selected along the time evolution. The number of distinct emitters selected during the interval $\kappa \delta t$ is extracted using the operator $M$, such that $M\left|\psi\left(t_{\mathcal{K}}\right)\right\rangle=m_{\mathcal{\kappa}}\left|\psi\left(t_{\kappa}\right)\right\rangle$, with $m_{\mathcal{K}}=$ $\sum_{n=1}^{N} \theta\left(\sum_{j=1}^{\mathcal{K}} \delta_{n, l_{j}}\right)$, where $\theta(x)$ is the step function $\theta(x>0)=1$, zero otherwise. Each eigenvalue $m_{\kappa}$ is $\Omega_{\kappa, m_{\kappa}}$ degenerated. There are two discrete symmetries that leave $M$ invariant: permutation of $\kappa$ elements along time and permutation of $N$ distinct objects. Both symmetries are expressed using the symmetric group $S_{\kappa} \otimes S_{N}$. Defining $\Omega_{\mathcal{K}}=\sum_{m=0}^{N} \Omega_{\kappa, m}$, the mean number of emitters in the ground state at time $t_{\kappa}$ is:

$$
\left\langle m_{\kappa}\right\rangle=\frac{1}{\Omega_{\kappa}} \sum_{m=0}^{N} m \Omega_{\kappa, m} .
$$

We stress that the analytic calculations of the degeneracy $\Omega_{\kappa, m}$ become increasingly harder as either $N$ or the time interval increases. Ultimately, the distribution $P_{n}(t)$ is the main goal, from which one can calculate or infer, for example, the instantaneous density of states or the instantaneous entropy $S(t)=-\sum_{n=0}^{N} P_{n}(t) \ln P_{n}(t)$. The values of $P_{n}(t)$ can be obtained either from statistical moments $\left\langle n^{\ell}(t)\right\rangle$ or from the instantaneous ratios $\Omega_{\kappa, n} / \Omega_{\kappa}$. However, it turns out that the calculation of $\Omega_{\kappa, m}$ can be simplified via a correspondence between the stochastic process and the classical birthday problem.

\section{The Birthday Problem}

Despite the hardships mentioned above, it is possible to craft a general solution using an elegant analogy with the classical process of drawing samples from an urn with $N$ distinct emitters. One at a time, an emitter is drawn from the urn; its label is recorded in a list, and then, it is replaced in the urn. This process is repeated $\kappa$ times. A sample is formed by the list of $\kappa$ recorded labels. In each sample, let $n$ be the number of distinct labels. Thus, $n=1, \ldots, \min (\kappa, N)$. We call $\Omega_{\kappa, n}^{\prime}$ the number of possible samples with $n$ different labels. 
This is precisely the formulation of the birthday problem, a set of $\kappa$ elements and uniform randomly-chosen tags are assigned out of a set of $N$ tags, with replacement, to each element. One concern is on the probability of having $n$ distinct tags (out of $N$ ) assigned to the $\kappa$ elements. There is a clear correspondence between the OTE configuration vectors in the limit $n \rightarrow m_{\kappa}$ and $\Omega_{\kappa, n}^{\prime} \rightarrow \Omega_{\kappa, m_{\kappa}}$. The number of ways $N$ distinct tags can be assigned to $\kappa$ elements is $N^{\kappa}$. One can group the $\kappa$ elements according to the $n$ distinct tags. This counting is given by the Stirling number of the second kind, which is non-vanishing for $n \geq 1$ and written as:

$$
\left\{\begin{array}{l}
\kappa \\
n
\end{array}\right\}=\frac{1}{n !} \sum_{j=0}^{n}(-1)^{j}\left(\begin{array}{l}
n \\
j
\end{array}\right)(n-j)^{\kappa} .
$$

Furthermore, the number of possible distinct $N$ tags in each group of size $n$ is given by the falling factorial power, which we represent by the Pochhammer symbol $(N)_{n}=N(N-1) \ldots(N-n+1)$. The number of possible ways of grouping $n$ distinct tags out of $N$ in $\kappa$ elements is precisely:

$$
\Omega_{\kappa, n}^{\prime}=(N)_{n}\left\{\begin{array}{l}
\kappa \\
n
\end{array}\right\}=\left(\begin{array}{l}
N \\
n
\end{array}\right) \sum_{j=0}^{n}(-1)^{j}\left(\begin{array}{l}
n \\
j
\end{array}\right)(n-j)^{\kappa} .
$$

In addition, the total number of groupings reduces to a far simpler expression:

$$
\Omega_{\kappa}^{\prime}=\sum_{n=0}^{\min (\kappa, N)} \Omega_{\kappa, n}^{\prime}=\sum_{n=0}^{\kappa}(N)_{n}\left\{\begin{array}{l}
\kappa \\
n
\end{array}\right\}=N^{\kappa},
$$

the upper limit in the summation can be taken to be $\kappa$ since $\left\{\begin{array}{l}\kappa \\ n\end{array}\right\}$ vanishes for $n>\kappa$.

The interpretation using the finite permutation group is very appealing. For a fixed $\kappa$, the $\ell^{\text {th }}$ statistical moment of $n$ is:

$$
\left\langle n^{\ell}\left(t_{\kappa}\right)\right\rangle=\frac{\sum_{n=0}^{\min (\kappa, N)} \Omega_{\kappa, n}^{\prime} n^{\ell}}{\sum_{n=0}^{\min (\kappa, N)} \Omega_{\kappa, n}^{\prime}}=\frac{1}{N^{\kappa}} \sum_{n=0}^{\kappa}(N)_{n}\left\{\begin{array}{l}
\kappa \\
n
\end{array}\right\} n^{\ell} .
$$

The degeneracy $\Omega_{\kappa, n}^{\prime}$ is required to evaluate higher statistical moments in both microscopic and OTE approaches. However, the OTE results are valid for arbitrary time intervals between consecutive events, whereas the microscopic approach requires the temporal differential equation. Thus, OTE can properly describe the rapid fluctuations that would not be captured otherwise during the transient. For $\ell=1$, one calculates that:

$$
\langle n(t)\rangle=N\left[1-\left(1-\frac{1}{N}\right)^{t}\right] \approx N\left(1-\mathrm{e}^{-t / N}\right),
$$

and for $\ell=2$,

$$
\frac{\left\langle n^{2}(t)\right\rangle}{N^{2}}=\frac{\langle n(t)\rangle}{N}-\left(1-\frac{1}{N}\right)^{t+1}\left[1-\left(\frac{1-2 / N}{1-1 / N}\right)\right] .
$$

Direct comparison between Equations (6) and (13) shows that both provide the same asymptotic $k=1$ statistical moment. However, there is a striking difference: the OTE description in Equation (13) is based on the symmetries of finite groups, and it is also valid for arbitrary time intervals, whereas Equation (6) requires constant and small time intervals $\delta t$.

Figure 2 shows the time evolution of scaled standard deviation $\sigma(t) / \sqrt{N}$, for $N / 32=$ $100,200,500,1000$, where all ensembles collapse under a single curve, dominated by the decay rate $\tau=2 N$ in discrete time. The standard deviation $\sigma(t)$ exhibits a maximum at $\left(t^{*} / N\right) \approx 1.3$, characterizing the rapid population change associated with the superradiant emission. However, these findings differ from the qualitative analysis of the Dicke model. In particular, $\left\langle n\left(t^{*}\right)\right\rangle \approx 0.73 N>N / 2$. 
Considering $\delta t=1 /\left(N p_{e}\right)$, since at most a single emission can occur within the time interval $\delta t$, the average emitted power is $P_{\text {avg }}=\hbar \omega\left\langle n\left(t^{*}\right)\right\rangle /\left(t^{*} \delta t\right) \propto \hbar \omega N p_{e}$, which fails to recover the $N^{2}$ behavior expected for superradiance. This can be explained by noting that the transitions in the Dicke model increases approximately with $n N$ until the maximum intensity is reached. Therefore, the simplification embedded in our model, that $p_{e}$ remains constant along the process, slows downs the cascade of emissions and reduces the power of emitted radiation.

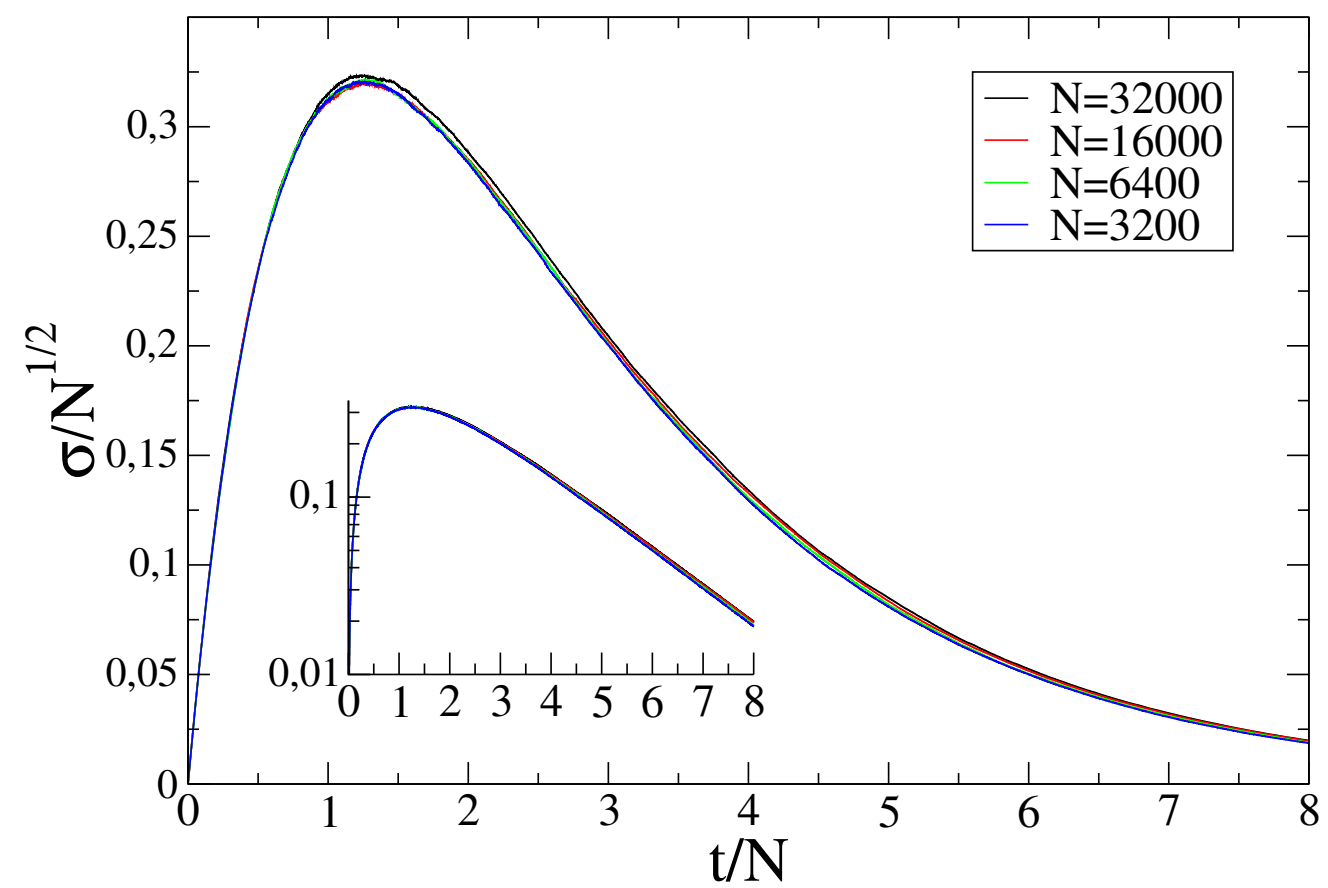

Figure 2. Time evolution of $\sigma(t) / \sqrt{N}$ using Monte Carlo simulations (50,000 runs). The natural time scale $t / N$ was employed so that the scaled standard deviation $\sigma(t / N) / \sqrt{N}$ collapses under a single shared curve, for small and large $N$. The inset (log scale) displays the long time dominant behavior, which has a characteristic decay time $\tau=2 N$.

\section{Conclusions}

We have shown that the OTE approach can be used to compute high-order statistics in self-organizing systems. The increased dimensional space permits the identification of finite symmetries and novel recurrence relations, which are used to evaluate high-order statistics required in superradiant systems. Despite these advances, we stress that the model considered in this study oversimplifies the superradiant dynamics by assuming a fixed conditional probability of emission $p_{e}=1$. An improved version of the model should contemplate $p_{e}$ as a function of $n$, or at least consider an upper value $p_{e}^{\text {upper }} \propto N$, to address the $N^{2}$ behavior of the radiation intensity in superradiance. Finally, we also mention that the method can also be used in other stochastic problems such as the disease spreading by aerial vectors. In vector-borne diseases, pathogens are transmitted between humans or from animals to humans through bloodsucking insects. Examples of those diseases are: Dengue fever, yellow fever, malaria, and leishmaniasis, just to cite a few. Analogous to the process of drawing samples from an urn, each bite can be seen as drawn from an urn (population) with $N$ distinct balls (hosts). Therefore, assuming that insect bites are randomly distributed among hosts, our result (Equation (6)) can be used to evaluate the statistical moments of this probabilistic system; where $k$ is the number of bites, $N$ the number of host individuals, and $n$ is the number infected hosts.

Author Contributions: A.S.M., B.C., and G.M.N. designed the research; B.C. and G.M.N. performed the research and wrote the computational codes; A.S.M. verified the numerical results; B.C. and G.M.N. wrote the paper; A.S.M., B.C., and G.M.N. edited the paper; all authors reviewed the manuscript. 
Funding: A.S.M. holds grants from CNPq 309851/2018-1. B.C. acknowledges Grant CAPES 88882.317469/2019-01. G.M.N. acknowledges Grant CAPES 88887.136416/2017-00.

Acknowledgments: We are grateful for César Augusto Terçariol and Tiago José Arruda for their helpful comments during the manuscript preparation.

Conflicts of Interest: The authors declare no conflict of interest.

\section{Abbreviations}

The following abbreviations are used in this manuscript:

DM Dicke model

SOP self-organizing phenomena

RWA rotating wave approximation

OTE ordered time evolution

\section{References}

1. Dicke, R.H. Coherence in Spontaneous Radiation Processes. Phys. Rev. 1954, 93, 99-110. [CrossRef]

2. Bohnet, J.G.; Chen, Z.; Weiner, J.M.; Meiser, D.; Holland, M.J.; Thompson, J.K. A steady-state superradiant laser with less than one intracavity photon. Nature 2012, 484, 78-81. [CrossRef] [PubMed]

3. Meiser, D.; Ye, J.; Carlson, D.R.; Holland, M.J. Prospects for a Millihertz-Linewidth Laser. Phys. Rev. Lett. 2009, 102, 163601. [CrossRef] [PubMed]

4. Meiser, D.; Holland, M.J. Intensity fluctuations in steady-state superradiance. Phys. Rev. A 2010, 81, 063827. [CrossRef]

5. Norcia, M.A.; Winchester, M.N.; Cline, J.R.K.; Thompson, J.K. Superradiance on the millihertz linewidth strontium clock transition. Sci. Adv. 2016, 2, [CrossRef] [PubMed]

6. Wang, D.W.; Liu, R.B.; Zhu, S.Y.; Scully, M.O. Superradiance Lattice. Phys. Rev. Lett. 2015, 114, 043602. [CrossRef] [PubMed]

7. Kloc, M.; Stránský, P.; Cejnar, P. Quantum quench dynamics in Dicke superradiance models. Phys. Rev. A 2018, 98, 013836. [CrossRef]

8. Scheibner, M.; Schmidt, T.; Worschech, L.; Forchel, A.; Bacher, G.; Passow, T.; Hommel, D. Superradiance of quantum dots. Nat. Phys. 2007, 3, 106-110. [CrossRef]

9. Mlynek, J.A.; Abdumalikov, A.A.; Eichler, C.; Wallraff, A. Observation of Dicke superradiance for two artificial atoms in a cavity with high decay rate. Nat. Commun. 2014, 5, 5186. [CrossRef]

10. Kozub, M.; Pawicki, L.; Machnikowski, P. Enhanced spontaneous optical emission from inhomogeneous ensembles of quantum dots is induced by short-range coupling. Phys. Rev. B 2012, 86, 121305. [CrossRef]

11. Abdussalam, W.; Machnikowski, P. Superradiance and enhanced luminescence from ensembles of a few self-assembled quantum dots. Phys. Rev. B 2014, 90, 125307. [CrossRef]

12. Yukalov, V.I.; Yukalova, E.P. Dynamics of quantum dot superradiance. Phys. Rev. B 2010, 81, 075308. [CrossRef]

13. Pustovit, V.N.; Shahbazyan, T.V. Cooperative emission of light by an ensemble of dipoles near a metal nanoparticle: The plasmonic Dicke effect. Phys. Rev. Lett. 2009, 102, 077401. [CrossRef]

14. Teperik, T.V.; Degiron, A. Superradiant Optical Emitters Coupled to an Array of Nanosize Metallic Antennas. Phys. Rev. Lett. 2012, 108, 147401. [CrossRef] [PubMed]

15. Yukalov, V.I. Origin of Pure Spin Superradiance. Phys. Rev. Lett. 1995, 75, 3000-3003. [CrossRef]

16. Yukalov, V.I.; Henner, V.K.; Kharebov, P.V.; Yukalova, E.P. Coherent spin radiation by magnetic nanomolecules and nanoclusters. Laser Phys. Lett. 2008, 5, 887. [CrossRef]

17. Yukalov, V.I.; Yukalova, E.P. Fast magnetization reversal of nanoclusters in resonator. J. Appl. Phys. $2012,111$. [CrossRef]

18. Yukalov, V.I.; Yukalova, E.P. Possibility of superradiance by magnetic nanoclusters. Laser Phys. Lett. 2011, 8, 804. [CrossRef]

19. Baumann, K.; Guerlin, C.; Brennecke, F.; Esslinger, T. Dicke quantum phase transition with a superfluid gas in an optical cavity. Nature 2010, 464, 1301-1306. [CrossRef] 
20. Klinder, J.; Keßler, H.; Wolke, M.; Mathey, L.; Hemmerich, A. Dynamical phase transition in the open Dicke model. Proc. Natl. Acad. Sci. 2015, 112, 3290-3295. [CrossRef]

21. Baumann, K.; Mottl, R.; Brennecke, F.; Esslinger, T. Exploring Symmetry Breaking at the Dicke Quantum Phase Transition. Phys. Rev. Lett. 2011, 107, 140402. [CrossRef] [PubMed]

22. Hepp, K.; Lieb, E.H. On the superradiant phase transition for molecules in a quantized radiation field: the Dicke maser model. Ann. Phys. (NY) 1973, 76, 360-404. [CrossRef]

23. Rotondo, P.; Cosentino Lagomarsino, M.; Viola, G. Dicke Simulators with Emergent Collective Quantum Computational Abilities. Phys. Rev. Lett. 2015, 114, 143601. [CrossRef] [PubMed]

24. Nicolis, G.; Prigogine, I. Symmetry breaking and pattern selection in far-from-equilibrium systems. Proc. Natl. Acad. Sci. USA 1981, 78, 659-663. [CrossRef] [PubMed]

25. Witten, T.A. Insights from soft condensed matter. Rev. Mod. Phys. 1999, 71, S367-S373. [CrossRef]

26. Cross, M.C.; Hohenberg, P.C. Pattern formation outside of equilibrium. Rev. Mod. Phys. 1993, 65, 851-1112. [CrossRef]

27. Lima, G.F.; Martinez, A.S.; Kinouchi, O. Deterministic Walks in Random Media. Phys. Rev. Lett. 2001, 87, 010603. [CrossRef]

28. Cabella, B.C.T.; Martinez, A.S.; Ribeiro, F. Data collapse, scaling functions, and analytical solutions of generalized growth models. Phys. Rev. E 2011, 83, 061902. [CrossRef]

29. Berbert, J.M.; González, R.S.; Martinez, A.S. Ergodic crossover in partially self-avoiding stochastic walks. Phys. Rev. E 2013, 88, 032119. [CrossRef]

30. Tavis, M.; Cummings, F.W. Exact Solution for an N-Molecule-Radiation-Field Hamiltonian. Phys. Rev. 1968, 170, 379-384. [CrossRef]

31. Emary, C.; Brandes, T. Quantum Chaos Triggered by Precursors of a Quantum Phase Transition: The Dicke Model. Phys. Rev. Lett. 2003, 90, 044101. [CrossRef] [PubMed]

32. Brandes, T. Excited-state quantum phase transitions in Dicke superradiance models. Phys. Rev. E 2013, 88, 032133. [CrossRef]

33. Chen, Q.H.; Zhang, Y.Y.; Liu, T.; Wang, K.L. Numerically exact solution to the finite-size Dicke model. Phys. Rev. A 2008, 78, 051801. [CrossRef]

34. Liu, T.; Zhang, Y.Y.; Chen, Q.H.; Wang, K.L. Large-N scaling behavior of the ground-state energy, fidelity, and the order parameter in the Dicke model. Phys. Rev. A 2009, 80, 023810. [CrossRef]

35. Rodriguez, J.P.J.; Chilingaryan, S.A.; Rodríguez-Lara, B.M. Critical phenomena in an extended Dicke model. Phys. Rev. A 2018, 98, 043805. [CrossRef]

36. Bastarrachea-Magnani, M.A.; Lerma-Hernández, S.; Hirsch, J.G. Thermal and quantum phase transitions in atom-field systems: a microcanonical analysis. J. Stat. Mech. Theory Exp. 2016, 2016, 093105. [CrossRef]

37. Gross, M.; Haroche, S. Superradiance: An essay on the theory of collective spontaneous emission. Phys. Rep. 1982, 93, 301-396. [CrossRef]

(C) 2019 by the authors. Licensee MDPI, Basel, Switzerland. This article is an open access article distributed under the terms and conditions of the Creative Commons Attribution (CC BY) license (http:/ / creativecommons.org/licenses/by/4.0/). 